\title{
Elusive transition to the ultimate regime of turbulent Rayleigh-Bénard convection
}

\author{
P. Urban, ${ }^{*}$ P. Hanzelka, T. Králík, M. Macek, and V. Musilová \\ Institute of Scientific Instruments, The Czech Academy of Sciences, Královopolská 147, Brno, Czech Republic \\ L. Skrbek ${ }^{\dagger}$ \\ Faculty of Mathematics and Physics, Charles University, Ke Karlovu 3, Prague, Czech Republic
}

(Received 5 June 2018; published 23 January 2019)

\begin{abstract}
By using cryogenic ${ }^{4} \mathrm{He}$ gas as the working fluid in a cylindrical cell $0.3 \mathrm{~m}$ in both height and diameter, we study the influence of non-Oberbeck-Boussinesq (NOB) effects on the heat transfer in turbulent RayleighBénard convection (RBC). We show that the NOB effects increase the heat transfer efficiency when the top plate temperature closely approaches the saturation vapor curve even far away from the critical point. Viewed in this light, our analysis points to the likelihood that the claim of having observed the transition to Kraichnan's ultimate regime, under nominally similar conditions in the experiments with $\mathrm{SF}_{6}$ [Phys. Rev. Lett. 108, 024502 (2012)], is probably an NOB effect and the important issue of the transition to the ultimate state of RBC remains open.
\end{abstract}

DOI: 10.1103/PhysRevE.99.011101

Turbulent convective flows occur on many length scales across the Universe. The equations describing the buoyancy driven flows and the corresponding convective heat transfer have been known for a long time [1,2], but our ability to predict flow behaviors for very intense convection occurring at large scales in the atmosphere, ocean, Sun, and the stars is quite limited. This is true even for the simplest model flow, the ideal laterally extended Rayleigh-Bénard convection (RBC) occurring in an Oberbeck-Boussinesq (OB) fluid layer [3] confined between two perfectly conducting plates, heated from below in a gravitational field. RBC is fully characterized by the Rayleigh number, $\mathrm{Ra}=g \frac{\alpha}{\nu \kappa} \Delta T L^{3}$, and the Prandtl number, $\operatorname{Pr}=v / \kappa$. One of the key issues here is the ability of RBC to transfer heat from the heated bottom plate to the cooled top plate, i.e., the convective heat transfer efficiency, described by the Nusselt number, $\mathrm{Nu}=\frac{L Q}{\lambda \Delta T}$, via the $\mathrm{Nu}=\mathrm{Nu}(\mathrm{Ra}$; $P r)$ dependence. Here, $Q$ is the total convective heat flux density, $g$ is the acceleration due to gravity, and $\Delta T=T_{\mathrm{b}}-T_{\mathrm{t}}$ is the temperature difference between the bottom and top plates separated by the vertical distance $L$. The working fluid is characterized by the thermal conductivity $\lambda$ and by the combination $\alpha /(\nu \kappa)=\eta$, where $\alpha$ is the isobaric thermal expansion, $v$ is the kinematic viscosity, and $\kappa$ denotes the thermal diffusivity. At very high $\mathrm{Ra}$, the $\mathrm{Nu}=\mathrm{Nu}(\mathrm{Ra} ; \mathrm{Pr})$ scaling for $\operatorname{Pr}=O(1)$ is usually expressed in the form of a scaling law $\mathrm{Nu} \propto \operatorname{Ra}^{\gamma} \operatorname{Pr}^{\beta}$. A plethora of exponents has been predicted $[1,2]$. Here, we mention two independent theories of Castaing et al. [4] and Shraiman and Siggia [5] predicting $\gamma=2 / 7$, while Priestley [6] and Malkus [7] derived $\gamma=1 / 3$.

This study is primarily concerned with experimental claims of the "ultimate" (or "asymptotic") regime, in which the RBC follows the prediction due to Kraichnan [8]. Kraichnan estimated that this regime would occur at a very high

\footnotetext{
*urban@isibrno.cz

†skrbek@fzu.cz
}

$\mathrm{Ra}^{*} \approx 10^{21}-10^{24}$, not yet achieved experimentally. He postulated it as the regime in which heat transfer becomes independent of $\kappa$ and $\nu$. When the convection reaches the ultimate regime, the boundary layers should be fully turbulent and the heat flux should become ballistic, leading to a scaling law of the form $\mathrm{Nu} \propto \mathrm{Ra}^{1 / 2} \mathrm{Pr}^{-1 / 4}(\log \mathrm{Ra})^{-3 / 2}$, predicted for $0.15<\operatorname{Pr}<1$ [for the "ultimate" $\mathrm{Nu} \propto(\operatorname{Pr} \mathrm{Ra})^{1 / 2}$ conjecture, see also Spiegel [9]]. It is hard to overestimate the importance of this asymptotic regime, a centerpiece of theoretical, numerical, and experimental studies of RBC at very high Ra. Observation of the transition to the ultimate state has been claimed several times in Grenoble experiments using cryogenic ${ }^{4} \mathrm{He}$ [10-12] and in the Göttingen experiments at ambient temperature using $\mathrm{SF}_{6}$ [13-15]. Here, we point out that the situation from an experimental point of view is likely to be open, just as it remains from a rigorous theoretical standpoint [16].

Experimentally (as well as numerically), RBC is often studied in cylindrical cells of diameter $D$ and height $L$; the relevant additional parameter is the aspect ratio $\Gamma=D / L$ [17]. In general, the physical properties of walls as well as of the surrounding medium, electric leads, mechanical support and its vibrations, tilt of plates, parasitic heat leaks, venting tubes, etc., affect the convective heat transfer which must therefore be generally studied via a functional dependence $\mathrm{Nu}=\mathrm{Nu}\left(\mathrm{Ra} ; \mathrm{Pr} ; \Gamma ; \mathrm{C}_{\text {expt }}^{0} \cdots \mathrm{C}_{\text {expt }}^{\mathrm{N}}\right)$, where $\mathrm{C}_{\text {expt }}^{0} \cdots \mathrm{C}_{\text {expt }}^{\mathrm{N}}$ are meant to address experimental details, leading to various corrections of the raw experimental data.

Following the 1975 pioneering experiment of Threlfall [18], and others that have followed it, we utilize the cryogenic ${ }^{4} \mathrm{He}$ gas, representing a working fluid with well-known properties [19] that are tunable in situ. The possibility of accessing a large range of $\mathrm{Ra}$, up to the highest attained in RBC laboratory experiments $\left(\approx 10^{17}\right)$ [20], is not the only advantage of using cryogenic ${ }^{4} \mathrm{He}$ gas. Cryogenic experiments benefit from an excellent thermal isolation because of the deep cryogenic vacuum surrounding the 
RBC cell. If placed inside a radiation shield thermally anchored at the helium bath, any parasitic heat leak due to the surrounding medium is simply not an issue. Further, favorable cryogenic properties of materials such as copper or stainless steel allow for the design of the RBC cell with a minimum influence of its structure on the convective flow [21]. The plates of our cell are made of 28 -mm-thick annealed oxygen-free high-conductivity copper (OFHC) of very high thermal conductivity (at least $2 \mathrm{~kW} \mathrm{~m}^{-1} \mathrm{~K}^{-1}$ at $5 \mathrm{~K}$ ). The thermal conductivity of the stainless steel sidewall (its thickness adjacent to plates where it matters most is only $0.5 \mathrm{~mm}$ ) is four orders of magnitude lower [22]. The thermal conductivity of plates is also higher than the actual convective heat transport enhanced by the turbulent Nusselt number. The cell is equipped with four newly calibrated (the best currently available precision being $\pm 2 \mathrm{mK}$ absolute accuracy over the entire temperature range of interest, PTB Berlin) Ge thermometers embedded in the middle and on the side of its copper plates. Additional sensors placed inside the cell, calibrated by us against the primary four, directly measure the turbulent core temperature $T_{\mathrm{c}}$. Contemporary electronics allow us to detect temperature differences of order $10 \mu \mathrm{K}$, with a sensitivity of about $10^{-5} T_{\mathrm{c}}$. With this level of confidence we see no horizontal temperature gradients in either copper plate.

We have shown [24] that for $7.2 \times 10^{6} \leqslant \mathrm{Ra} \leqslant 10^{11}$ our sidewall-corrected data agree with suitably corrected data from complementary cryogenic experiments, and are consistent with $\mathrm{Nu} \propto \mathrm{Ra}^{2 / 7}$. On approaching $\mathrm{Ra} \approx 10^{11}$, all cryogenic data display a broad crossover to $\mathrm{Nu} \propto \mathrm{Ra}^{1 / 3}$, as predicted by Refs. [6,7], and in accord with the theoretical model of Grossmann and Lohse [25] and its update by Stevens et al. [26]. The existing claim is that, for $\mathrm{Ra}>10^{12}$, a transition to an ultimate regime occurs and that, upon further increasing Ra, the scaling exponent $\gamma$ starts to exceed $1 / 3$. This is one reason why we have chosen the range $2 \times 10^{12} \lesssim$ $\mathrm{Ra} \lesssim 3 \times 10^{13}$ for our experiment. Additional reasons for this choice are as follows: (i) Corrections due to the walls, important at smaller Ra, are already small here; (ii) corrections due to plates are not yet important; (iii) $\Delta T$ between the plates can be set much bigger than that due to the adiabatic temperature gradient (up to about $1 \mathrm{mK}$ ) arising due to the hydrostatic pressure of the working fluid in the cell; and (iv) the conventional $\mathrm{OB}$ criterion $\alpha \Delta T \leqslant$ const $\cong 0.2$ can be satisfied.

All the above considerations implicitly assumed that the working fluid can be treated as an OB fluid [3]. In practice, the $\mathrm{OB}$ conditions are never fully satisfied, especially for laboratory experiments performed with gaseous working fluids in the vicinity of their critical points (CPs) and saturated vapor curves (SVCs), in order to achieve high Ra. In this Rapid Communication, we focus on measurements near the SVC. Here, the vicinity of the first-order (vapor-liquid) phase transition represents a much milder problem to the OB conditions (unless the SVC line is actually crossed) compared to the vicinity of $\mathrm{CP}$, where the working fluid properties diverge (e.g., the specific heat $C_{P}$ ) or vanish (e.g., the thermal diffusivity $\kappa$ ). Our major point is that serious consequences occur when one works in the vicinity of SVC, and that it needs to be carefully addressed.
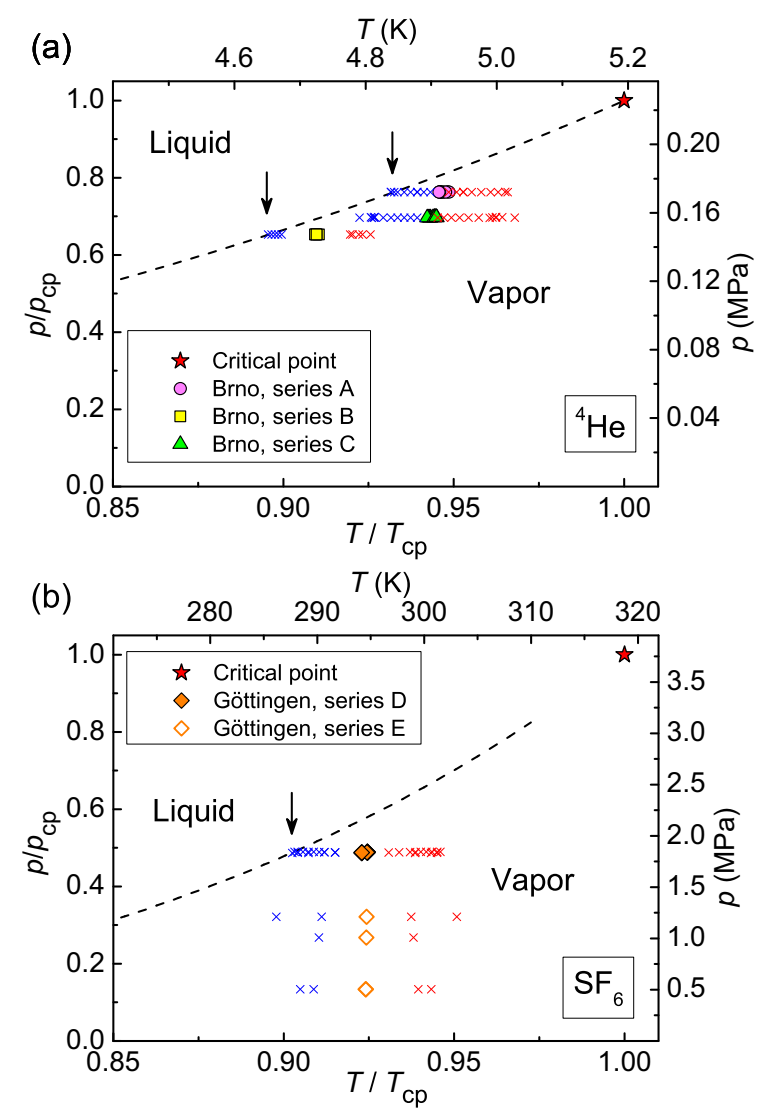

FIG. 1. (a) ${ }^{4} \mathrm{He}$ and (b) $\mathrm{SF}_{6} p$ - $T$ phase diagrams, showing their critical points $\left(T_{\mathrm{cp}}, p_{\mathrm{cp}}\right)$ and SVCs (dashed lines). The color-coded symbols (labeled in the inset) denote the experimental working points $(p, T)$ in ${ }^{4} \mathrm{He}$ and $\mathrm{SF}_{6}$, which are approximately equally distant from the $\mathrm{CP}$ in dimensionless phase diagrams. The $\mathrm{SF}_{6}$ data are adapted from Ref. [14]. The data series discussed in the text is traceable via the pairs of corresponding temperatures $T_{\mathrm{b}}($ red $\times)$ and $T_{\mathrm{t}}($ blue $\times$ ) as measured in particular experimental runs. The arrows show the series which operate close to SVC.

We discuss here three series of heat transfer measurements performed in our Brno laboratory using ${ }^{4} \mathrm{He}$, labeled A, B, and $\mathrm{C}$ in Fig. 1(a), and compare them with an analogous series of measurements, labeled D and E in Fig. 1(b), performed in Göttingen using the $\mathrm{SF}_{6}$ gas, in which the transition to the ultimate regime was recently claimed [13]. The Brno series A-C was obtained at three (approximately constant) values of pressure $p$, with $0.02<\alpha \Delta T<0.18$, which spans about a decade in Ra. In each series, $\Delta T$ was sequentially increased so that the top (bottom) plate temperatures $T_{t}\left(T_{b}\right)$ approached (departed from) the SVC, by adjusting and monitoring the respective cooling (heating) rates. The protocols and the positions with respect to SVC (and CP) are very similar to those of the Göttingen $\mathrm{SF}_{6}$ measurements, as is readily seen in Fig. 1, where the $p$ and $T$ axes are labeled in units relative to the critical points of ${ }^{4} \mathrm{He}$ [Fig. 1(a)] and $\mathrm{SF}_{6}$ [Fig. 1(b)].

The quantities $Q, \Delta T=T_{\mathrm{b}}-T_{\mathrm{t}}, T_{\mathrm{c}}, T_{\mathrm{m}}=\left(T_{\mathrm{b}}+T_{\mathrm{t}}\right) / 2$, together with the known fluid properties of ${ }^{4} \mathrm{He}$ [19], allow us to calculate the boundary layer asymmetry and heat transfer efficiency, via the Nusselt (Nu) versus Ra scaling, where both $\mathrm{Nu}$ and $\mathrm{Ra}$ could be evaluated in various ways attempting to 


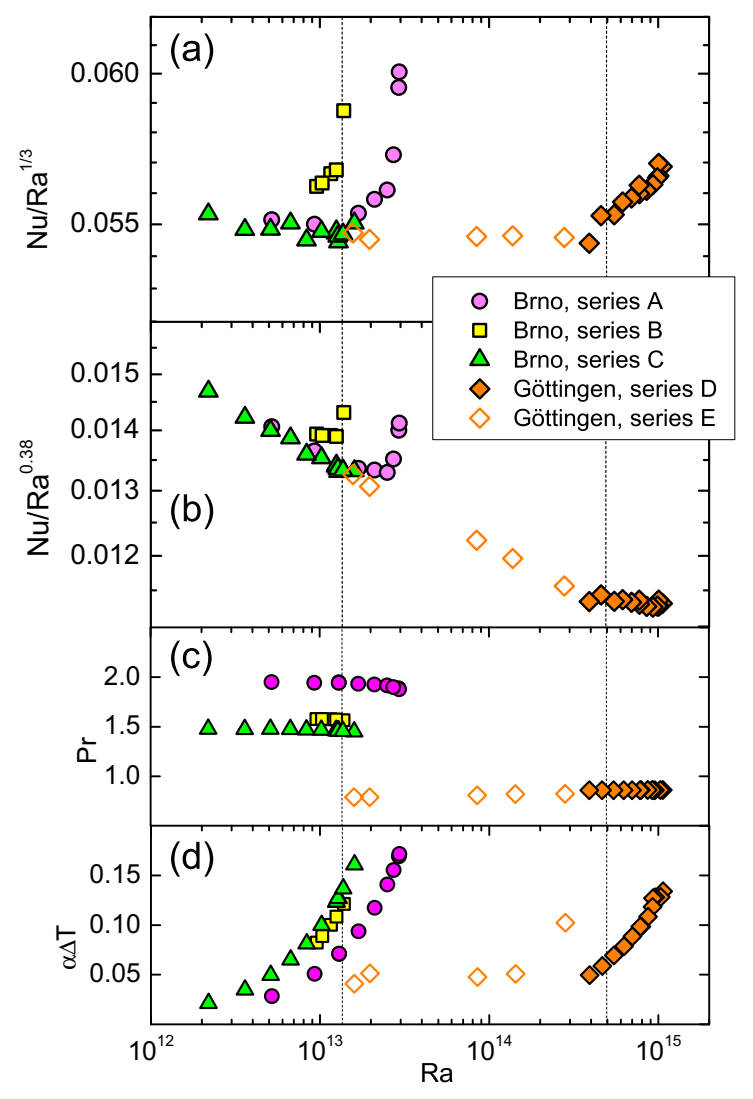

FIG. 2. (a) Compensated $\mathrm{Nu} / \mathrm{Ra}^{1 / 3}$ and (b) $\mathrm{Nu} / \mathrm{Ra}^{0.38}$ vs $\mathrm{Ra}$ plots of the Brno data (series A, B, and C) shown together with the Göttingen data (series D and E) [13], obtained under nominally similar conditions. Note the departure from the $\mathrm{Nu} \propto \mathrm{Ra}^{1 / 3}$ scaling for the data points with $T_{\mathrm{t}}$ lying in close vicinity of SVC, marked by arrows in the corresponding $p$ - $T$ phase diagrams shown in Fig. 1. These data points, with $T_{\mathrm{t}}$ still in the gas phase (see Fig. 3), display a "transition" to $\mathrm{Nu} \propto \mathrm{Ra}^{0.38}$, claimed as effectively ultimate scaling $[13,33]$, while the last three data points of series $A$ and the last point of series B, with $T_{\mathrm{t}}$ already in the liquid side, indicate an abrupt increase in heat transfer efficiency due to condensation and evaporation in the boundary layer [34], not present in the $\mathrm{SF}_{6}$ data. In contrast, the controlled Brno series $\mathrm{C}$ as well as $\mathrm{SF}_{6}$ data points [13] that correspond to the lowest Ra with $T_{\mathrm{t}}$ farther away from the SVC (marked as series E in Fig. 1) follow the $\mathrm{Ra}^{1 / 3}$ scaling (top). The two lower panels show (c) the Prandtl number and (d) $\alpha \Delta T$ evaluated at $\alpha\left(T_{\mathrm{m}}\right)$ for the same data.

correct for the non-Oberbeck-Boussinesq (NOB) effects, as described earlier by us in detail [27-29]. Here, for clarity and simplicity, we calculate the $\mathrm{Nu}(\mathrm{Ra})$ scaling conventionally, i.e., based on directly measured $Q, \Delta T$, and the fluid properties evaluated at the measured $p$ and $T_{\mathrm{m}}=\left(T_{\mathrm{b}}+T_{\mathrm{t}}\right) / 2$, and show the resulting compensated $\mathrm{Nu}(\mathrm{Ra})$ scaling in Fig. 2 .

The central result of this Rapid Communication is shown in Fig. 2. The data series $\mathrm{A}$ and $\mathrm{B}$, for which $T_{\mathrm{t}}$ closely approaches SVC, displays a strong increase of the $\mathrm{Nu}(\mathrm{Ra})$ slope [Figs. 2(a) and 2(b)] around Ra of order $10^{13}$. In the case of series $\mathrm{B}$, the change occurs outside the range suggested for the transition to the ultimate regime claimed by He et al. [13] (see also the more detailed publications [14,30]), shown by vertical dotted lines here in Fig. 2. In striking contrast, the
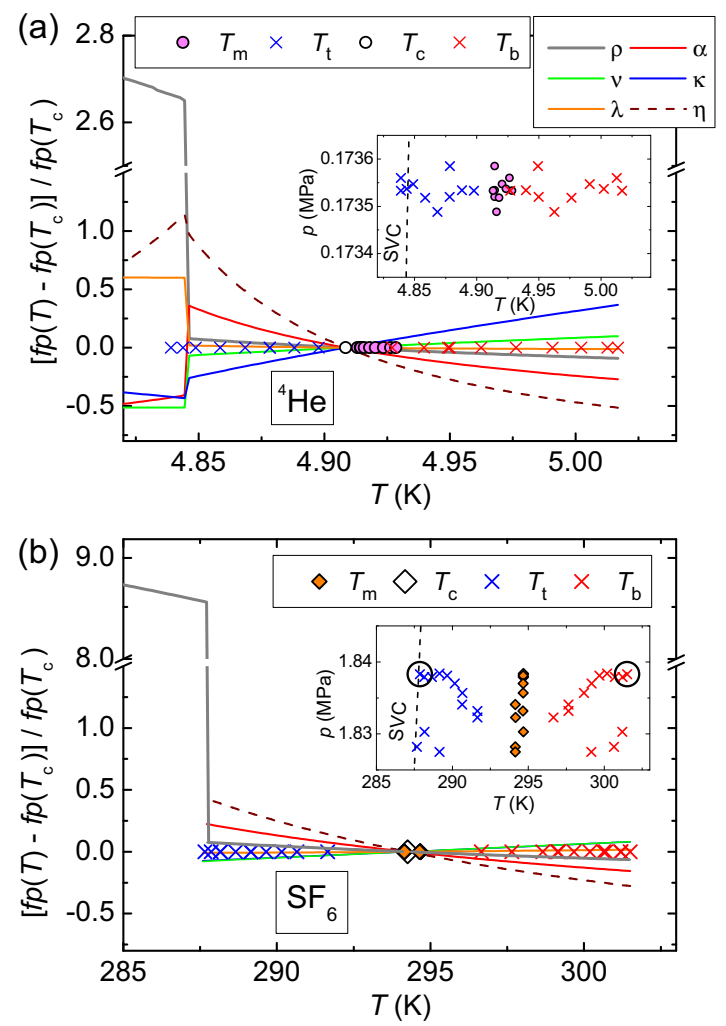

FIG. 3. Temperature dependencies of the relevant physical properties $f p$ evaluated for measurements in ${ }^{4} \mathrm{He}$ [(a) series $\left.\mathrm{A}\right]$ and $\mathrm{SF}_{6}$ [(b) series D; the $T_{\mathrm{t}}$ and $T_{\mathrm{b}}$ temperatures corresponding to the maximum-Ra measurement are highlighted by circles], relative to the $f p$ values at respective $T_{\mathrm{c}}$. The insets show that the scatter of pressure values is (a) $\delta p / p \lesssim 0.05 \%$ and (b) $\lesssim 0.5 \%$, respectively, and confirm that all the Göttingen $\mathrm{SF}_{6}$ data lie in the gaseous side of the SVC [inset (b)], while the top plate temperatures for three ${ }^{4} \mathrm{He}$ data points of series A lie on the liquid side of the SVC [inset (a)]. Due to asymmetry of boundary layers, the calculated $T_{\mathrm{m}}$ temperatures gradually shift with increasing $\Delta T$, while the directly measured center temperatures $T_{\mathrm{c}}$ stay constant (within experimental accuracy). The directly measured top (bottom) plate temperatures are indicated by red (blue) crosses. The SVC is marked by a jump in the density $\rho$.

controlled series $\mathrm{C}$, with $T_{\mathrm{t}}$ farther away from the SVC, closely follows the $\mathrm{Ra}^{1 / 3}$ scaling. Our data from series $\mathrm{C}$ agree with the Göttingen series E [13,14], measured far away from the SVC of $\mathrm{SF}_{6}$, as seen in Fig. 1. This confirms the high precision of both experiments and also the sufficient accuracy with which the fluid properties of ${ }^{4} \mathrm{He}[19]$ and $\mathrm{SF}_{6}[31,32]$ are known far away from SVC.

Figure 2(c) shows that the Prandtl number $\mathrm{Pr} \cong 1$ for all data sets displayed. The observed increase in the $\mathrm{Nu}(\mathrm{Ra})$ slope in all displayed data sets therefore cannot be associated with variable Pr. Let us add that our earlier NOB data [27-29] clearly displayed a strong increase in the $\mathrm{Nu}(\mathrm{Ra})$ slope for $\mathrm{Ra}>10^{14}$ for even higher Prandtl numbers $3 \lesssim$ $\operatorname{Pr} \lesssim 16$, while no such tendency was observed by Niemela et al. up to $10^{17}$ in $\mathrm{Ra}$ [20]. Still, all these data satisfy the commonly used OB criterion $\alpha \Delta T \leqslant 0.2$ [see Fig. 2(d)]. This clearly indicates that this criterion is not sufficient and the 
fluid properties must be explored in more detail, as done below. In particular, the currently discussed "near SVC case" should be distinguished from the more complex "near CP case" of Refs. [10-12,20,27-29].

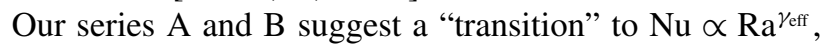
i.e., to ultimate scaling characterized by the effective scaling exponent $\gamma_{\text {eff }} \approx 0.38$, and may be interpreted to support the claims by Ref. [13] and to agree with the prediction of Grossman and Lohse [33]. We emphasize that as these "transitions" occur at different $\mathrm{Ra}^{*}$ and under experimental conditions which cannot be treated as OB, they cannot be interpreted as the transition to Kraichnan's ultimate state [8]. Additionally, our data with $T_{\mathrm{t}}$ already on the liquid phase of ${ }^{4} \mathrm{He}$ SVC indicate yet another abrupt increase in heat transfer efficiency due to condensation and evaporation in the boundary layer, in accord with the experiments of Zhong et al. [34], and that this increase in slope is much steeper than the "ultimate scaling."

In Fig. 3 we plot the relevant fluid properties for the Brno ${ }^{4} \mathrm{He}$ data of series A [19] [Fig. 3(a)] and of Göttingen $\mathrm{SF}_{6}$ [31] data of series D [Fig. 3(b)]. The asymmetry of the boundary layers (somewhat stronger for series A) is readily visible. Clearly, if the measured $T_{\mathrm{c}}$ (nearly the same in the entire bulk of the turbulent core) stays approximately constant, the calculated $T_{\mathrm{m}}$ slides towards higher temperatures as a consequence of this asymmetry. Both fluids display significant NOB features. Moreover, the fluid properties are not constant upon approaching the SVC, where they change faster in a nonlinear fashion. While $\lambda$ changes only slightly with temperature, the combination $\alpha /(\nu \kappa)=\eta$ that enters the definition of Ra does so appreciably. Consequently, the thicknesses of the top and bottom boundary layers significantly differ (assuming them to be marginally stable in the range of $\mathrm{Ra}$ where $\mathrm{Nu} \propto \mathrm{Ra}^{1 / 3}$ ). Note that the conventional OB criterion, written in the form $\alpha \Delta T \ll 1$, simply means the incompressibility condition in the boundary layers over which most of $\Delta T$ occurs. Evaluating $\alpha$ for this criterion as $\alpha\left(T_{\mathrm{m}}\right)$ or $\alpha\left(T_{\mathrm{c}}\right)$ therefore does not adequately characterize the boundary layer flow in the case of temperature-dependent $\alpha(T)$.

It is possible that, even if one took account of the gradual nonlinear change of equilibrium NOB fluid properties in the proximity of the equilibrium SVC, it might not be enough to fully resolve this issue-a possibility of a transition in the boundary layer ought to be considered [35], as it could trigger a transition to a more effectively conducting state of turbulent convection, but this might not necessarily require a change in slope of the Nu-Ra plot.

To conclude, our results unequivocally demonstrate that for the correct evaluation of heat transfer efficiency of turbulent $\mathrm{RBC}$ at high $\mathrm{Ra}$ one ought to take into account the NOB effects that arise when $T_{\mathrm{t}}$ closely approaches the ${ }^{4} \mathrm{He}$ SVC from the gaseous side of the $p-T$ phase diagram, even if the operating points are far away from the critical point. Considering the NOB effects is crucial for all experiments aiming to observe the transition to the ultimate regime of RBC. In particular, our analysis points to the likelihood that the crossover to the ultimate regime [13], based on experimental points with $T_{\mathrm{t}}$ highlighted by an arrow in the $\mathrm{SF}_{6}$ phase diagram [see Fig. 1(b)], is most likely caused by the NOB effects in the $\mathrm{SF}_{6}$ used in the close vicinity of the SVC. Thus, the important and intriguing question of the transition to the ultimate state of RBC (see also Refs. [20,27-29]) remains open.

This research is funded by the Czech Science Foundation under Project GAČR 17-03572S. L.S. appreciates stimulating discussions with K. R. Sreenivasan; M.M. acknowledges funding by EU structural and investment funds and MEYS CR OPVVV Project No. CZ.02.2.69/0.0/0.0/18 070/0009944 (qCULTURA).
[1] G. Ahlers, S. Grossmann, and D. Lohse, Rev. Mod. Phys. 81, 503 (2009).

[2] F. Chillà and J. Schumacher, Eur. Phys. J. E 35, 58 (2012).

[3] The OB fluid has constant physical properties except its density which linearly depends on temperature.

[4] B. Castaing, G. Gunaratne, F. Heslot, L. Kadanoff, A. Libchaber, S. Thomae, X.-Z. Wu, S. Zaleski, and G. Zanetti, J. Fluid Mech. 204, 1 (1989).

[5] B. I. Shraiman and E. D. Siggia, Phys. Rev. A 42, 3650 (1990).

[6] C. H. B. Priestley, Turbulent Transfer in the Lower Atmosphere (University of Chicago Press, Chicago, 1959).

[7] M. V. R. Malkus, Proc. R. Soc. London, Ser. A 225, 196 (1954).

[8] R. H. Kraichnan, Phys. Fluids 5, 1374 (1962).

[9] E. A. Spiegel, Annu. Rev. Astron. Astrophys. 9, 323 (1971).

[10] X. Chavanne, F. Chillà, B. Castaing, B. Hébral, B. Chabaud, and J. Chaussy, Phys. Rev. Lett. 79, 3648 (1997).

[11] X. Chavanne, F. Chillà, B. Chabaud, B. Castaing, and B. Hébral, Phys. Fluids 13, 1300 (2001).

[12] P.-E. Roche, F. Gauthier, R. Kaiser, and J. Salort, New J. Phys. 12, 085014 (2010).

[13] X. He, D. Funfschilling, H. Nobach, E. Bodenschatz, and G. Ahlers, Phys. Rev. Lett. 108, 024502 (2012).
[14] G. Ahlers, X. He, D. Funfschilling, and E. Bodenschatz, New J. Phys. 14, 103012 (2012).

[15] Note, however, that in Ref. [11] the authors of Ref. [13] dispute the claim of observing the transition to the ultimate state of RBC in the Grenoble experiments.

[16] C. R. Doering, Rigorous results for heat transport in high Rayleigh number convection, presented at the International Conference on RB Turbulence, Hong Kong, December 10-14, 2012 (unpublished).

[17] Experimental (as well as theoretical or computational) studies performed in finite aspect ratio cells (especially for $\Gamma<1$ ) cannot be understood as representing the general behavior of laterally infinite RBC even if the container walls were ideal, as a new length scale, the lateral size $D$, is introduced to the RBC flow under study.

[18] D. C. Threlfall, J. Fluid Mech. 67, 17 (1975).

[19] R. D. McCarty, National Bureau of Standards Technical Note No. 631, 1972; V. D. Arp and R. D. McCarty, The properties of critical helium gas, Technical Report, University of Oregon, 1998.

[20] J. J. Niemela, L. Skrbek, K. R. Sreenivasan, and R. J. Donnelly, Nature (London) 404, 837 (2000). 
[21] P. Urban, P. Hanzelka, T. Kralik, V. Musilova, L. Skrbek, and A. Srnka, Rev. Sci. Instrum. 81, 085103 (2010).

[22] One way to estimate the influence of the sidewall is via the socalled wall parameter defined by Roche and co-workers [23]; our cell has the lowest value out of all so far used cryogenic cells.

[23] P.-E. Roche, B. Castaing, B. Chabaud, B. Hébral, and J. Sommeria, Eur. Phys. J. B 24, 405 (2001).

[24] P. Urban, V. Musilová, and L. Skrbek, Phys. Rev. Lett. 107, 014302 (2011).

[25] S. Grossmann and D. Lohse, Phys. Rev. E 66, 016305 (2002).

[26] R. J. A. M. Stevens, E. P. van der Poel, S. Grossmann, and D. Lohse, J. Fluid Mech. 730, 295 (2013).

[27] P. Urban, P. Hanzelka, T. Kralik, V. Musilova, A. Srnka, and L. Skrbek, Phys. Rev. Lett. 109, 154301 (2012).

[28] P. Urban, P. Hanzelka, V. Musilová, T. Králík, M. L. Mantia, A. Srnka, and L. Skrbek, New J. Phys. 16, 053042 (2014).
[29] L. Skrbek and P. Urban, J. Fluid Mech. 785, 270 (2015).

[30] X. He, D. Funfschilling, E. Bodenschatz, and G. Ahlers, New J. Phys. 14, 063030 (2012).

[31] To evaluate the $\mathrm{SF}_{6}$ properties we use the computer program of the Göttingen group, kindly provided to us by G. Ahlers and X. $\mathrm{He}$, together with the NIST database https://www.nist.gov/srd/ refprop.

[32] C. Guder and W. Wagner, J. Phys. Chem. Ref. Data 38, 33 (2009).

[33] S. Grossmann and D. Lohse, Phys. Fluids 23, 045108 (2011).

[34] J-Q. Zhong, D. Funfschilling, and G. Ahlers, Phys. Rev. Lett. 102, 124501 (2009).

[35] F. Gauthier and P.-E. Roche, Europhys. Lett. 83, 24005 (2008); F. Gauthier, J. Salort, O. Bourgeois, J.-L. Garden, R. du Puits, A. Thess, and P.-E. Roche, ibid. 87, 44006 (2009). 